Pacific

Journal of

Mathematics

\title{
INFINITE-DIMENSIONAL SANDWICH PAIRS
}

MARTIN SCHECHTER 


\title{
INFINITE-DIMENSIONAL SANDWICH PAIRS
}

\author{
MARTIN SCHECHTER
}

\begin{abstract}
For many equations arising in the physical sciences, the solutions are critical points of functionals. This has led to interest in finding critical points of such functionals. If a functional $G$ is semibounded, one can find PalaisSmale (PS) sequences $G\left(u_{k}\right) \rightarrow a$ and $G^{\prime}\left(u_{k}\right) \rightarrow 0$. These sequences produce critical points if they have convergent subsequences (that is, if $G$ satisfies the PS condition). However, there is no clear method of finding critical points of functionals that are not semibounded. In this paper we find pairs of sets having the property that functionals bounded from below on one set and bounded from above on the other have PS sequences. We can allow both sets to be infinite-dimensional if we make a slight additional smoothness requirement on the functional. This allows us to solve systems of equations that could not be solved before.
\end{abstract}

\section{Introduction}

Many problems arising in science and engineering call for the solving of the Euler equations of functionals, that is, equations of the form

$$
G^{\prime}(u)=0,
$$

where $G(u)$ is a $C^{1}$ functional (usually with physical dimension of energy) arising from the given data. As an illustration, the equation $-\Delta u(x)=f(x, u(x))$ is the Euler equation of the functional

$$
G(u)=\frac{1}{2}\|\nabla u\|^{2}-\int F(x, u(x)) d x
$$

on an appropriate space, where

$$
F(x, t)=\int_{0}^{t} f(x, s) d s,
$$

and the norm is that of $L^{2}$. Solving the Euler equations is tantamount to finding critical points of the corresponding functional. The classical approach was to look

MSC2000: $35 \mathrm{~J} 65,58 \mathrm{E} 05,49 \mathrm{~J} 35$.

Keywords: critical point theory, linking, variational methods, saddle point theory, sandwich pairs, semilinear partial differential equations, critical sequences. 
for maxima or minima. If one is looking for a minimum, it is not sufficient to know that the functional is bounded from below, as is easily observed. If it is bounded from below, one can obtain a minimizing sequence satisfying

$$
G\left(u_{k}\right) \rightarrow a=\inf G
$$

If such a sequence has a convergent subsequence, then we indeed obtain a minimum. However, in dealing with such sequences it is difficult, in general, to establish the convergence of a subsequence.

Luckily, there is some help. One can show that there is a sequence, called a Palais-Smale (PS) sequence satisfying

$$
G\left(u_{k}\right) \rightarrow a \quad \text { and } \quad G^{\prime}\left(u_{k}\right) \rightarrow 0,
$$

where $a=\inf G$. It is much easier to establish the existence of a convergent subsequence of a PS sequence than of a minimizing sequence. In fact, a minimizing sequence may not have a convergent subsequence while a PS sequence for the same functional does. If every PS sequence for $G$ has a convergent subsequence, then we say that $G$ satisfies the PS condition.

However, when the functional is not semibounded, there is no clear way of obtaining critical points. Is there anything that can be used to replace semiboundedness? Expressed otherwise, how can one level the playing field? One approach is called linking. As a substitute for semiboundedness, one can look for suitable sets that separate the given functional, that is, suitable subsets $A$ and $B$ of the space $E$ satisfying

$$
a_{0}:=\sup _{A} G \leq b_{0}:=\inf _{B} G
$$

for a given $C^{1}$ functional $G$ on $E$. There are pairs of subsets such that (1) produces a PS sequence

$$
G\left(u_{k}\right) \rightarrow a \quad \text { and } \quad G^{\prime}\left(u_{k}\right) \rightarrow 0,
$$

where $a \geq b_{0}$. If $A$ and $B$ are such that (1) always implies (2), we say that $A$ links $B$. Consequently, if $A$ links $B$ and $G$ is a $C^{1}$ functional on $E$ that satisfies (1) and the PS condition, then $G$ has a critical point satisfying

$$
G(u)=a \geq b_{0} \quad \text { and } \quad G^{\prime}(u)=0 .
$$

Linking sets exist and are described in the literature; see for example, [Schechter 1999].

In [Schechter 2008] we discussed the situation in which one cannot find linking sets that separate the functional, that is, that satisfy (1). Are there sets such that the 
opposite of (1) will imply (2)? More precisely, are there sets $A$ and $B$ such that

$$
-\infty<b_{0}:=\inf _{B} G \quad \text { and } \quad a_{0}:=\sup _{A} G<\infty
$$

implies that there is a sequence satisfying

$$
G\left(u_{k}\right) \rightarrow c \quad \text { for } b_{0} \leq c \leq a_{0} \quad \text { and } \quad G^{\prime}\left(u_{k}\right) \rightarrow 0 ?
$$

This was answered in the affirmative. Such pairs exist. This has led to

Definition 1. We say that a pair of subsets $A$ and $B$ of a Banach space $E$ forms a sandwich, if for any $G \in C^{1}(E, \mathbb{R})$ the inequality (3) implies the existence of a PS sequence (4).

The root of this approach comes from the work of Silva [1991] and the author [1993; 1992]. They proved

Theorem 2. Let $N$ be a closed subspace of a Hilbert space E, and let $M=N^{\perp}$. Assume that at least one of the subspaces $M$ and $N$ is finite-dimensional. Let $G$ be a $C^{1}$-functional on $E$ such that

$$
m_{0}:=\inf _{w \in M} G(w) \neq-\infty \quad \text { and } \quad m_{1}:=\sup _{v \in N} G(v) \neq \infty .
$$

Then there are a constant $c \in \mathbb{R}$ and a sequence $\left\{u_{k}\right\} \subset E$ such that

$$
G\left(u_{k}\right) \rightarrow c \quad \text { with } m_{0} \leq c \leq m_{1} \quad \text { and } \quad G^{\prime}\left(u_{k}\right) \rightarrow 0 .
$$

This theorem, called the "sandwich theorem", is very useful in dealing with equations or systems for which the corresponding functional is semibounded in one of the directions only on a subspace of finite dimension. However, there are many systems for which this is not the case. On the other hand, the theorem is probably not true if both subspaces are infinite-dimensional.

Here, we shall show that the theorem is indeed true if we require a bit more than mere continuous differentiability of the functional. The requirement we have chosen is present in many applications.

Definition 3. Let $E$ be a Banach space. We shall call a functional $G \in C^{1}(E, \mathbb{R})$ weak-to-weak continuously differentiable if for each sequence

$$
u_{k} \rightarrow u \text { weakly in } E
$$

there exists a renamed subsequence such that

$$
G^{\prime}\left(u_{k}\right) \rightarrow G^{\prime}(u) \text { weakly. }
$$

For such functionals we have 
Theorem 4. Let $N$ be a closed subspace of a Hilbert space $E$ and let $M=N^{\perp}$. Let $G$ be a weak-to-weak continuously differentiable functional on $E$ such that

$$
m_{0}:=\inf _{w \in M} G(w) \neq-\infty \quad \text { and } \quad m_{1}:=\sup _{v \in N} G(v) \neq \infty .
$$

Then there are a constant $c \in \mathbb{R}$ and a sequence $\left\{u_{k}\right\} \subset E$ such that

$$
G\left(u_{k}\right) \rightarrow c \quad \text { with } m_{0} \leq c \leq m_{1} \quad \text { and } \quad G^{\prime}\left(u_{k}\right) \rightarrow 0 .
$$

We shall prove Theorem 4 in Section 2, where we introduce weak sandwich pairs. Applications will be given in Section 3. One purpose of our investigation is to solve systems of equations of the form

$$
\mathbb{A} v=f(x, v, w) \quad \text { and } \quad \mathbb{B} w=g(x, v, w),
$$

where $\mathbb{A}$ and $\mathbb{B}$ are linear partial differential operators. Such systems have many applications. For instance, they can describe multiple chemical reactions or stable states of dynamical systems determined by reaction diffusion equations.

Unlike linking, the order of a sandwich pair is immaterial, that is, if the pair $A, B$ forms a sandwich, so does $B, A$. Moreover, we allow sets forming a sandwich pair to intersect. (A description of sandwich pairs can be found in [Schechter 2008].) It follows from Theorem 2 that $M$ and $N$ form a sandwich pair if one of them is finite-dimensional. (Note that $m_{0} \leq m_{1}$.) This is a severe drawback in many applications.

The purpose of the present paper is find a counterpart of sandwich pairs that deals with the case when both sets in the pair are infinite-dimensional. To do this we require weak-to-weak continuous differentiability of the functional as we did in Theorem 4. We call such pairs weak sandwich pairs.

\section{Weak sandwich pairs}

We now introduce the corresponding definition for the case when both sets $A$ and $B$ are infinite-dimensional.

Definition 5. We shall say that a pair of subsets $A$ and $B$ of a Banach space $E$ forms a weak sandwich pair, if for any weak-to-weak continuously differentiable $G \in C^{1}(E, \mathbb{R})$ the inequality

$$
-\infty<b_{0}:=\inf _{B} G \leq a_{0}:=\sup _{A} G<\infty
$$

implies that there is a sequence $\left\{u_{k}\right\}$ satisfying

$$
G\left(u_{k}\right) \rightarrow c \quad \text { with } b_{0} \leq c \leq a_{0} \quad \text { and } \quad G^{\prime}\left(u_{k}\right) \rightarrow 0 .
$$


Theorem 6. Let $E$ be a separable Hilbert space, let $N$ be a closed subspace of $E$, and let $p$ be any point of $N$. Let $F$ be a Lipschitz continuous map of $E$ onto $N$ such that $\left.F\right|_{N}=I$ and $\|F(g)-F(h)\| \leq K\|g-h\|$ for $g, h \in E$. Suppose also that for each finite-dimensional subspace $S$ of $E$ containing $p$ such that $F S \neq\{0\}$, there is a finite-dimensional subspace $S_{0} \neq\{0\}$ of $N$ containing $p$ such that $v \in S_{0}$ and $w \in S$ implies $F(v+w) \in S_{0}$. (The stipulation that $S_{0} \neq\{0\}$ is made in case $p=0$.) Then $A=N$ and $B=F^{-1}(p)$ form a weak sandwich pair.

Proof. Assume that the theorem is false. Let $G$ be a weak-to-weak continuously differentiable functional on $E$ satisfying (6), where $A$ and $B$ are the subsets of $E$ specified in the theorem, such that there is no sequence satisfying (7). Then there is a positive number $\delta>0$ such that

$$
\left\|G^{\prime}(u)\right\| \geq 2 \delta
$$

whenever $u$ belongs to the set $\hat{E}=\left\{\in E: b_{0}-2 \delta \leq G(u) \leq a_{0}+2 \delta\right\}$. Since $E$ is separable, we can norm it with a norm $|u|_{w}$ satisfying $|u|_{w} \leq\|u\|$ for $u \in E$ and such that the topology induced by this norm is equivalent to the weak topology of $E$ on bounded subsets of $E$.

This can be done as follows. Let $\left\{e_{k}\right\}$ be an orthonormal basis for $E$. We set

$$
|u|_{w}^{2}=\sum_{k=1}^{\infty} \frac{\left|\left(u, e_{k}\right)\right|^{2}}{k^{2}} .
$$

We denote $E$ equipped with this norm by $\tilde{E}$. For $u \in \hat{E}$, let $h(u)=G^{\prime}(u) /\left\|G^{\prime}(u)\right\|$. Then by (8)

$$
\left(G^{\prime}(u), h(u)\right) \geq 2 \delta \quad \text { for } u \in \hat{E} .
$$

Let

$$
\begin{array}{ll}
T=\left(a_{0}-b_{0}+4 \delta\right) / \delta, & B_{R}=\{u \in E:\|u\|<R\}, \\
R=\sup _{\Omega}\|u\|+T, & \hat{B}=\bar{B}_{R} \cap \hat{E},
\end{array}
$$

where $\Omega$ is a bounded open subset of $N$ containing the point $p$ such that $\rho(\partial \Omega, p)>$ $K T+\delta$ and $\rho$ is the distance in $E$. For each $u \in \hat{B}$ there is an $\tilde{E}$ neighborhood $W(u)$ of $u$ such that $\left(G^{\prime}(v), h(u)\right)>\delta$ for $v \in W(u) \cap \hat{B}$. For otherwise there would be a sequence $\left\{v_{k}\right\} \subset \hat{B}$ such that

$$
\left|v_{k}-u\right|_{w} \rightarrow 0 \quad \text { and } \quad\left(G^{\prime}\left(v_{k}\right), h(u)\right) \leq \delta .
$$

Since $\hat{B}$ is bounded in $E, v_{k} \rightarrow u$ weakly in $E$ and (5) implies that

$$
\left(G^{\prime}\left(v_{k}\right), h(u)\right) \rightarrow\left(G^{\prime}(u), h(u)\right) \leq \delta
$$


in view of (10). This contradicts (9). Let $\tilde{B}$ be the set $\hat{B}$ with the inherited topology of $\tilde{E}$. It is a metric space, and $W(u) \cap \tilde{B}$ is an open set in this space. Thus, $\{W(u) \cap \tilde{B}\}$ for $u \in \tilde{B}$ is an open covering of the paracompact space $\tilde{B}$ (see for example [Kelley 1955]). Consequently, there is a locally finite refinement $\left\{W_{\tau}\right\}$ of this cover. For each $\tau$ there is an element $u_{\tau}$ such that $W_{\tau} \subset W\left(u_{\tau}\right)$. Let $\left\{\psi_{\tau}\right\}$ be a partition of unity subordinate to this covering. Each $\psi_{\tau}$ is locally Lipschitz continuous with respect to the norm $|u|_{w}$ and consequently with respect to the norm of $E$. Let $Y(u)=\sum \psi_{\tau}(u) h\left(u_{\tau}\right)$ for $u \in \tilde{B}$. Then $Y(u)$ is locally Lipschitz continuous with respect to both norms. Moreover,

$$
\|Y(u)\| \leq \sum \psi_{\tau}(u)\left\|h\left(u_{\tau}\right)\right\| \leq 1
$$

and

$$
\left(G^{\prime}(u), Y(u)\right)=\sum \psi_{\tau}(u)\left(G^{\prime}(u), h\left(u_{\tau}\right)\right) \geq \delta \quad \text { for } u \in \hat{B} .
$$

For $u \in \bar{\Omega} \cap \hat{E}$, let $\sigma(t) u$ be the solution of

$$
\sigma^{\prime}(t)=-Y(\sigma(t)) \text { for } t \geq 0 \text { and } \sigma(0)=u .
$$

Note that $\sigma(t) u$ will exist as long as $\sigma(t) u$ is in $\hat{B}$. Also, it is continuous in $(u, t)$ with respect to both topologies.

Next we note that if $u \in \bar{\Omega} \cap \hat{E}$ we cannot have $\sigma(t) u \in \hat{B}$ and $G(\sigma(t) u)>b_{0}-\delta$ for $0 \leq t \leq T$. For by (13) and (12),

$$
d G(\sigma(t) u) / d t=\left(G^{\prime}(\sigma), \sigma^{\prime}\right)=-\left(G^{\prime}(\sigma), Y(\sigma)\right) \leq-\delta
$$

as long as $\sigma(t) u \in \hat{B}$. Hence if $\sigma(t) u \in \hat{B}$ for $0 \leq t \leq T$, we would have

$$
G(\sigma(T) u)-G(u) \leq-\delta T=-\left(a_{0}-b_{0}+4 \delta\right) .
$$

Thus, we would have $G(\sigma(T) u)<b_{0}-4 \delta$. On the other hand, $\sigma(s) u$ exists for $0 \leq s<T$. To see this note that

$$
u-\sigma(t) u=z_{t}(u):=\int_{0}^{t} Y(\sigma(s) u) d s .
$$

By (11), we have $\left\|z_{t}(u)\right\| \leq t$. Consequently, $\|\sigma(t) u\| \leq\|u\|+t<R$. Thus $\sigma(t) u \in \hat{B}$. We can now conclude that for each $u \in \bar{\Omega} \cap \hat{E}$ there is a $t \geq 0$ such that $\sigma(s) u$ exists for $0 \leq s \leq t$ and $G(\sigma(t) u) \leq b_{0}-\delta$. Let

$$
T_{u}:=\inf \left\{t \geq 0: G(\sigma(t) u) \leq b_{0}-\delta\right\} \quad \text { for } u \in \bar{\Omega} \cap \hat{E} .
$$

Then $\sigma(t) u$ exists for $0 \leq t \leq T_{u}$ and $T_{u}<T$, and $T_{u}$ is continuous in $u$. Define

$$
\hat{\sigma}(t) u=\left\{\begin{array}{ll}
\sigma(t) u & \text { if } 0 \leq t \leq T_{u}, \\
\sigma\left(T_{u}\right) u & \text { if } T_{u} \leq t \leq T,
\end{array} \quad \text { for } u \in \bar{\Omega} \cap \hat{E} .\right.
$$


For $u \in \bar{\Omega} \backslash \hat{E}$, define $\hat{\sigma}(t) u=u$ for $0 \leq t \leq T$. Then $\hat{\sigma}(t) u$ is continuous in $(u, t)$, and

$$
G(\hat{\sigma}(T) u) \leq b_{0}-\delta \quad \text { for } u \in \bar{\Omega}
$$

Let

$$
\varphi(v, t)=F \hat{\sigma}(t) v \quad \text { for } v \in \bar{\Omega} \quad \text { and } \quad 0 \leq t \leq T .
$$

Then $\varphi$ is a continuous map of $\bar{\Omega} \times[0, T]$ to $N$. Let

$$
K=\{(u, t): u=\hat{\sigma}(t) v \text { for } v \in \bar{Q} \text { and } t \in[0, T]\} .
$$

Then $K$ is a compact subset of $\tilde{E} \times \mathbb{R}$. To see this, let $\left(u_{k}, t_{k}\right)$ be any sequence in $K$. Then $u_{k}=\sigma\left(t_{k}\right) v_{k}$, where $v_{k} \in \bar{Q}$. Since $Q$ is bounded, there is a subsequence such that $v_{k} \rightarrow v_{0}$ weakly in $E$ and $t_{k} \rightarrow t_{0}$ in $[0, T]$. Since $\bar{Q}$ is convex and bounded, $v_{0}$ is in $\bar{Q}$ and $\left|v_{k}-v_{0}\right|_{w} \rightarrow 0$. Since $\hat{\sigma}(t)$ is continuous in $\tilde{E} \times \mathbb{R}$,

$$
u_{k}=\hat{\sigma}\left(t_{k}\right) v_{k} \rightarrow \hat{\sigma}\left(t_{0}\right) v_{0}=u_{0} \in K .
$$

Each $u_{0} \in \hat{B}$ has a neighborhood $W\left(u_{0}\right)$ in $\tilde{E}$ and a finite-dimensional subspace $S\left(u_{0}\right)$ such that $Y(u) \subset S\left(u_{0}\right)$ for $u \in W\left(u_{0}\right) \cap \hat{B}$. Since $\hat{\sigma}(t) u$ is continuous in $(u, t)$, for each $\left(u_{0}, t_{0}\right) \in K$ there is a neighborhood $W\left(u_{0}, t_{0}\right) \subset \tilde{E} \times \mathbb{R}$ and a finite-dimensional subspace $S\left(u_{0}, t_{0}\right) \subset E$ such that $\hat{z}_{t}(u) \subset S\left(u_{0}, t_{0}\right)$ for $(u, t) \in$ $W\left(u_{0}, t_{0}\right)$, where

$$
\hat{z}_{t}(u):=u-\hat{\sigma}(t) u= \begin{cases}\int_{0}^{t} Y(\hat{\sigma}(s) u) d s & \text { if } u \in \hat{E}, \\ 0 & \text { if } u \notin \hat{E} .\end{cases}
$$

Since $K$ is compact, there is a finite number of points $\left(u_{j}, t_{j}\right) \subset K$ such that $K \subset W=\bigcup W\left(u_{j}, t_{j}\right)$. Let $S$ be a finite-dimensional subspace of $E$ containing $p$ and all the $S\left(u_{j}, t_{j}\right)$ and such that $F S \neq\{0\}$. Then for each $v \in \bar{\Omega}$, we have $\hat{z}_{t}(v) \in S$. Then by hypothesis, there is a finite-dimensional subspace $S_{0} \neq\{0\}$ of $N$ containing $p$ such that $F\left(v-\hat{z}_{t}(v)\right) \in S_{0}$ for all $v \in \bar{\Omega} \cap S_{0}$. We note that $\varphi(u, t)$ maps $\bar{\Omega} \cap S_{0} \times[0, T]$ into $S_{0}$. For $t$ in $[0, T]$, let $\varphi_{t}(v)=\varphi(v, t)$. Then

$$
\varphi_{t}(v) \neq p \quad \text { for } v \in \partial\left(\Omega \cap S_{0}\right)=\partial \Omega \cap S_{0} \quad \text { and } \quad 0 \leq t \leq T .
$$

To see this, note that if $v \in \partial \Omega$, then $\|v-p\| \leq\|v-F \hat{\sigma}(t) v\|+\|F \hat{\sigma}(t) v-p\|$. Hence $\|F \hat{\sigma}(t) v-p\|>K T+\delta-t K>0$ for $v \in \partial \Omega$ and $\leq t \leq T$, since

$$
\|F \hat{\sigma}(t) v-v\| \leq K \int_{0}^{t}\left\|\hat{\sigma}^{\prime}(s) v\right\| d s \leq K t .
$$


Thus (16) holds. Consequently the Brouwer degree $d\left(\varphi_{t}, \Omega \cap S_{0}, p\right)$ is defined. Since $\varphi_{t}$ is continuous, we have

$$
d\left(\varphi_{T}, \Omega \cap S_{0}, p\right)=d\left(\varphi_{0}, \Omega \cap S_{0}, p\right)=d\left(I, \Omega \cap S_{0}, p\right)=1 .
$$

Hence there is a $v \in \Omega$ such that $F \hat{\sigma}(T) v=p$. Consequently $\hat{\sigma}(T) v \in F^{-1}(p)=B$. In view of (6), this implies $G(\hat{\sigma}(T) v) \geq b_{0}$, contradicting (14). Thus (7) holds, and the proof is complete.

Proof of Theorem 4. We take $A=N, B=M, p=0$, and $F=P_{N}$, the projection onto $N$. If $S$ is a finite-dimensional subspace such that $F S \neq\{0\}$, we take $S_{0}=F S$. All of the hypotheses of Theorem 6 are satisfied.

Definition 7. Let $E$ and $F$ be Banach spaces. We shall call a map $J \in C(E, F)$ weak-to-weak continuous if for each sequence

$$
u_{k} \rightarrow u \text { weakly in } E
$$

there exists a renamed subsequence such that

$$
J\left(u_{k}\right) \rightarrow J(u) \text { weakly in } F .
$$

Proposition 8. If $A$ and $B$ is a weak sandwich pair and $J$ is a weak-to-weak continuous diffeomorphism on the entire space having a derivative $J^{\prime}(u)$ depending compactly on $u$ and satisfying

$$
\left\|J^{\prime}(u)^{-1}\right\| \leq C \quad \text { for } u \in E,
$$

then $J A$ and $J B$ is a weak sandwich pair.

Proof. Let $G$ be a weak-to-weak continuously differentiable functional on $E$ satisfying

$$
-\infty<b_{0}:=\inf _{J B} G \leq a_{0}:=\sup _{J A} G<\infty .
$$

Let $G_{1}(u)=G(J u)$ for $u \in E$. Then $\left(G_{1}(u), h\right)=\left(G^{\prime}(J u), J^{\prime}(u) h\right)$. If $u_{k} \rightarrow u$ weakly, then there is a renamed subsequence such that $J\left(u_{k}\right) \rightarrow J(u)$ weakly and $J^{\prime}\left(u_{k}\right) \rightarrow J^{\prime}(u)$. Hence, $\left(G_{1}\left(u_{k}\right), h\right) \rightarrow\left(G^{\prime}(J u), J^{\prime}(u) h\right)$, and $G_{1}$ is weak-to-weak continuously differentiable. Moreover,

$$
\begin{aligned}
-\infty<b_{0} & :=\inf _{J B} G=\inf _{J u \in J B} G(J u)=\inf _{B} G_{1} \\
\leq a_{0} & :=\sup _{J A} G=\sup _{J u \in J A} G(J u)=\sup _{A} G_{1}<\infty .
\end{aligned}
$$

Since $A$ and $B$ form a weak sandwich pair, there is a sequence $\left\{h_{k}\right\} \subset E$ such that

$$
G_{1}\left(h_{k}\right) \rightarrow c \quad \text { for } b_{0} \leq c \leq a_{0} \quad \text { and } \quad G_{1}^{\prime}\left(h_{k}\right) \rightarrow 0 .
$$


If we set $u_{k}=J h_{k}$, this becomes $G\left(u_{k}\right) \rightarrow c$ for $b_{0} \leq c \leq a_{0}$ and $G^{\prime}\left(u_{k}\right) J^{\prime}\left(h_{k}\right) \rightarrow 0$. In view of (17), this implies $G^{\prime}\left(u_{k}\right) \rightarrow 0$. Thus $J A$ and $J B$ form a weak sandwich pair.

Proposition 9. Let $N$ be a closed subspace of a Hilbert space $E$ with complement $M^{\prime}=M \oplus\left\{v_{0}\right\}$, where $v_{0}$ is an element in $E$ having unit norm, and let $\delta$ be any positive number. Let $\varphi(t) \in C^{1}(\mathbb{R})$ be such that $0 \leq \varphi(t) \leq 1, \varphi(0)=1$, and $\varphi(t)=0$ for $|t| \geq 1$. Let

$$
F\left(v+w+s v_{0}\right)=v+\left[s+\delta-\delta \varphi\left(\|w\|^{2} / \delta^{2}\right)\right] v_{0} \quad \text { for } \quad v \in N, w \in M, s \in \mathbb{R} .
$$

Then $A=N^{\prime}=N \oplus\left\{v_{0}\right\}$ and $B=F^{-1}\left(\delta v_{0}\right)$ form a weak sandwich pair.

Proof. Define

$J\left(v+w+s v_{0}\right)=v+w+\left[s-\delta+\delta \varphi\left(\|w\|^{2} / \delta^{2}\right)\right] v_{0} \quad$ for $\quad v \in N, w \in M, s \in \mathbb{R}$.

Then $J$ is a diffeomorphism on $E$ satisfying the hypotheses of Proposition 8. Also, $A=J N^{\prime}$ and $B=J\left[M+\delta v_{0}\right]$. Since $N^{\prime}$ and $M+\delta v_{0}$ form a weak sandwich pair by Theorem 4, $A$ and $B$ also form a weak sandwich pair (Proposition 8).

\section{Applications}

Let $A$ and $B$ be positive, self-adjoint operators on $L^{2}(\Omega)$ with compact resolvents, where $\Omega \subset \mathbb{R}^{n}$. Let $F(x, v, w)$ be a Carathéodory function on $\Omega \times \mathbb{R}^{2}$ such that

$$
f(x, v, w)=\partial F / \partial v \quad \text { and } \quad g(x, v, w)=\partial F / \partial w
$$

are also Carathéodory functions satisfying

$$
|f(x, v, w)|+|g(x, v, w)| \leq C_{0}(|v|+|w|+1) \quad \text { for } v, w \in \mathbb{R}
$$

and

$$
\begin{aligned}
& f(x, t y, t z) / t \rightarrow \alpha_{+}(x) v^{+}-\alpha_{-}(x) v^{-}+\beta_{+}(x) w^{+}-\beta_{-}(x) w^{-}, \\
& g(x, t y, t z) / t \rightarrow \gamma_{+}(x) v^{+}-\gamma_{-}(x) v^{-}+\delta_{+}(x) w^{+}-\delta_{-}(x) w^{-}
\end{aligned}
$$

as $t \rightarrow+\infty, y \rightarrow v$, and $z \rightarrow w$, where $a^{ \pm}=\max ( \pm a, 0)$. We wish to solve the system

$$
\begin{aligned}
\mathbb{A} v & =-f(x, v, w), \\
\mathbb{B} w & =g(x, v, w) .
\end{aligned}
$$

Such systems have been studied in the literature by many authors (for example, [Costa 1994; Costa and Magalhães 1994; de Figueiredo and Felmer 1994; Furtado and Silva 2001; Furtado et al. 2002a; 2002b; Li and Yang 2004; Schechter 1998; Schechter and Zou 2003; Silva 2001; Tintarev 1999; Peihao et al. 2002; Zou 2001] 
and the literature quoted in them). Let $\lambda_{0}\left(\mu_{0}\right)$ be the lowest eigenvalue of $\mathbb{A}(\mathbb{B})$. We assume that the only solution of

$$
\begin{aligned}
-\mathbb{A} v & =\alpha_{+} v^{+}-\alpha_{-} v^{-}+\beta_{+} w^{+}-\beta_{-} w^{-}, \\
\mathbb{B} w & =\gamma_{+} v^{+}-\gamma_{-} v^{-}+\delta_{+} w^{+}-\delta_{-} w^{-}
\end{aligned}
$$

is $v=w=0$.

The equations (21) take the place of the equation characterizing the Fučík spectrum for a problem involving only one function. Essentially, our hypotheses require that $\left(\alpha_{+}, \alpha_{-}, \beta_{+}, \beta_{-}, \gamma_{+}, \gamma_{-}, \delta_{+}, \delta_{-}\right)$is not in the "Fučík" spectrum of $(-\mathbb{A}, \mathbb{B})$.

Our first result is

Theorem 10. Assume

$$
\begin{array}{llll}
2 F(x, s, 0) \geq-\lambda_{0} s^{2}-W_{1}(x) & \text { for } x \in \Omega & \text { and } & t \in \mathbb{R}, \\
2 F(x, 0, t) \leq \mu_{0} t^{2}+W_{2}(x) & \text { for } x \in \Omega & \text { and } & t \in \mathbb{R},
\end{array}
$$

where $W_{i}(x) \in L^{1}(\Omega)$. Then the system (20) has a solution.

Proof. Let $D=D\left(\mathbb{A}^{1 / 2}\right) \times D\left(\mathbb{B}^{1 / 2}\right)$. Then $D$ becomes a Hilbert space with norm given by $\|u\|_{D}^{2}=(\mathbb{A} v, v)+(\mathbb{B} w, w)$ for $u=(v, w) \in D$. We define

$$
G(u)=b(w)-a(v)-2 \int_{\Omega} F(x, v, w) d x \quad \text { for } u \in D,
$$

where $a(v)=(\mathbb{A} v, v)$ and $b(w)=(\mathbb{B} w, w)$. Then $G \in C^{1}(D, \mathbb{R})$ and

$$
\left(G^{\prime}(u), h\right) / 2=b\left(w, h_{2}\right)-a\left(v, h_{1}\right)-\left(f(u), h_{1}\right)-\left(g(u), h_{2}\right),
$$

where we write $f(u)$ and $g(u)$ in place of $f(x, v, w)$ and $g(x, v, w)$, respectively. It is readily seen that the system (20) is equivalent to

$$
G^{\prime}(u)=0 .
$$

We let $N$ be the set of those $(v, 0) \in D$, and let $M$ be the set of those $(0, w) \in D$. Then $M$ and $N$ are orthogonal closed subspaces such that $D=M \oplus N$. If we define $L u=2(-v, w)$ for $u=(v, w) \in D$, then $L$ is a selfadjoint bounded operator on $D$. Also $G^{\prime}(u)=L u+c_{0}(u)$, where $c_{0}(u)=-\left(\mathbb{A}^{-1} f(u), \mathbb{B}^{-1} g(u)\right)$ is compact on $D$. This follows from (18) and the fact that $\mathbb{A}$ and $\mathbb{B}$ have compact resolvents. It also follows that $G^{\prime}$ has weak-to-weak continuity. For if $u_{k} \rightarrow u$ weakly, then $L u_{k} \rightarrow L u$ weakly and $c_{0}\left(u_{k}\right)$ has a convergent subsequence. Now by (23)

$$
G(0, w) \geq b(w)-\mu_{0}\|w\|^{2}-\int_{\Omega} W_{2}(x) d x \quad \text { for }(0, w) \in M .
$$

Thus

$$
\inf _{M} G \geq-\int_{\Omega} W(x) d x \equiv b_{0} .
$$


On the other hand, (22) implies

$$
G(v, 0) \leq-a(v)+\lambda_{0}\|v\|^{2}+\int_{\Omega} W_{1}(x) d x \quad \text { for }(v, 0) \in N .
$$

Thus

$$
\sup _{N} G \leq \int_{\Omega} W_{1}(x) d x \equiv a_{0} .
$$

We can now apply Theorem 4 to conclude that there is a sequence $\left\{u_{k}\right\} \subset D$ such that (7) holds. Let $u_{k}=\left(v_{k}, w_{k}\right)$. I claim that

$$
\rho_{k}^{2}=a\left(v_{k}\right)+b\left(w_{k}\right) \leq C .
$$

For assume that $\rho_{k} \rightarrow \infty$, and let $\tilde{u}_{k}=u_{k} / \rho_{k}$. Then there is a renamed subsequence such that $\tilde{u}_{k} \rightarrow \tilde{u}$ weakly in $D$, strongly in $L^{2}(\Omega)$ and almost everywhere in $\Omega$. If $h=\left(h_{1}, h_{2}\right) \in D$, then

$$
\left(G^{\prime}\left(u_{k}\right), h\right) / \rho_{k}=2 b\left(\tilde{w}_{k}, h_{2}\right)-2 a\left(\tilde{v}_{k}, h_{1}\right)-2\left(f\left(u_{k}\right), h_{1}\right) / \rho_{k}-2\left(g\left(u_{k}\right), h_{2}\right) / \rho_{k} .
$$

Taking the limit and applying (18) and (19), we see that $\tilde{u}=(\tilde{v}, \tilde{w})$ is a solution of (21). Hence $\tilde{u}=0$ by hypothesis. On the other hand, since $a\left(\tilde{v}_{k}\right)+b\left(\tilde{w}_{k}\right)=1$, there is a renamed subsequence such that $a\left(\tilde{v}_{k}\right) \rightarrow \tilde{a}$ and $b\left(\tilde{w}_{k}\right) \rightarrow \tilde{b}$ with $\tilde{a}+\tilde{b}=1$. Thus by (19) and (24)

$$
\begin{aligned}
\left(G^{\prime}\left(u_{k}\right),\left(\tilde{v}_{k}, 0\right)\right) / 2 \rho_{k} & =-a\left(\tilde{v}_{k}\right)-\left(f\left(u_{k}\right), \tilde{v}_{k}\right) / \rho_{k} \\
& \rightarrow-\tilde{a}-\int_{\Omega}\left(\alpha_{+} \tilde{v}^{+}-\alpha_{-} \tilde{v}^{-}+\beta_{+} \tilde{w}^{+}-\beta_{-} \tilde{w}^{-}\right) \tilde{v} d x, \\
\left(G^{\prime}\left(u_{k}\right),\left(0, \tilde{w}_{k}\right)\right) / 2 \rho_{k} & =b\left(\tilde{w}_{k}\right)-\left(g\left(u_{k}\right), \tilde{w}_{k}\right) / \rho_{k} \\
& \rightarrow \tilde{b}-\int_{\Omega}\left(\gamma_{+} \tilde{v}^{+}-\gamma_{-} \tilde{v}^{-}+\delta_{+} \tilde{w}^{+}-\delta_{-} \tilde{w}^{-}\right) \tilde{w} d x .
\end{aligned}
$$

Thus by (7),

$$
\begin{aligned}
& \tilde{a}=-\int_{\Omega}\left(\alpha_{+} \tilde{v}^{+}-\alpha_{-} \tilde{v}^{-}+\beta_{+} \tilde{w}^{+}-\beta_{-} \tilde{w}^{-}\right) \tilde{v} d x, \\
& \tilde{b}=\int_{\Omega}\left(\gamma_{+} \tilde{v}^{+}-\gamma_{-} \tilde{v}^{-}+\delta_{+} \tilde{w}^{+}-\delta_{-} \tilde{w}^{-}\right) \tilde{w} d x .
\end{aligned}
$$

Since one of the two numbers $\tilde{a}$ and $\tilde{b}$ is not zero, we cannot have $\tilde{u} \equiv 0$. This contradiction proves (27). This known, we can use the usual procedures to show that there is a renamed subsequence such that $u_{k} \rightarrow u$ in $D$, and $u$ satisfies (25).

Theorem 11. In addition, assume that the eigenfunctions of $\lambda_{0}$ and $\mu_{0}$ are bounded and $\neq 0$ almost everywhere in $\Omega$, and there is a $q>2$ such that $\|w\|_{q}^{2} \leq C b(w)$ for $w \in M$. Assume $2 F(x, 0, t) \leq \mu(x) t^{2}$ for $x \in \Omega$ and $t \in \mathbb{R}$, where 


$$
\begin{aligned}
\mu(x) & \leq \mu_{0} \quad \text { and } \quad \mu(x) \not \equiv \mu_{0} \quad \text { for } x \in \Omega, \\
2 F(x, s, t) & \leq \mu_{0} t^{2}-\lambda_{0} s^{2} \quad \text { for }|t|+|s| \leq \delta,
\end{aligned}
$$

for some $\delta>0$. Then the system (20) has a nontrivial solution.

Proof. Let $N^{\prime}$ be the orthogonal complement of $N_{0}=\left\{\left(\varphi_{0}, 0\right)\right\}$ in $N$, where $\varphi_{0}$ is the eigenfunction of $\mathbb{A}$ corresponding to $\lambda_{0}$. Then $N=N^{\prime} \oplus N_{0}$. Let $M_{0}$ be the subspace of $M$ spanned by the eigenfunctions $\{(0, \psi)\}$ of $B$ corresponding to $\mu_{0}$, and let $M^{\prime}$ be its orthogonal complement in $M$. Since $N_{0}$ and $M_{0}$ are contained in $L^{\infty}(\Omega)$, there is a positive constant $\rho$ such that

$$
\begin{array}{ll}
a(y) \leq \rho^{2} \Rightarrow\|y\|_{\infty} \leq \delta / 4 & \text { for } y \in N_{0}, \\
b(h) \leq \rho^{2} \Rightarrow\|h\|_{\infty} \leq \delta / 4 & \text { for } h \in M_{0},
\end{array}
$$

where $\delta$ is the number in (29). If $a(y) \leq \rho^{2}, b(w) \leq \rho^{2}$, and $|y(x)|+|w(x)| \geq \delta$, we write $w=h+w^{\prime}, h \in M_{0}, w^{\prime} \in M^{\prime}$, and

$$
\delta \leq|y(x)|+|w(x)| \leq|y(x)|+|h(x)|+\left|w^{\prime}(x)\right| \leq(\delta / 2)+\left|w^{\prime}(x)\right| .
$$

Thus

$$
\begin{aligned}
& |y(x)|+|h(x)| \leq \delta / 2 \leq\left|w^{\prime}(x)\right|, \\
& |y(x)|+|w(x)| \leq 2\left|w^{\prime}(x)\right| .
\end{aligned}
$$

Now by (29) and (31)

$$
\begin{aligned}
G(y, w) & =b(w)-a(y)-2 \int_{\Omega} F(x, y, w) d x \\
& \geq b(w)-a(y)-\int_{|y|+|w|<\delta}\left(\mu_{0} w^{2}-\lambda_{0} y^{2}\right) d x-c_{0} \int_{|y|+|w|>\delta}(|y|+|w|+1)^{2} d x \\
& \geq b(w)-a(y)-\mu_{0}\|w\|^{2}+\lambda_{0}\|y\|^{2}-c_{1} \int_{2\left|w^{\prime}\right|>\delta}\left|w^{\prime}\right|^{q} d x \\
& \geq b\left(w^{\prime}\right)-\mu_{0}\left\|w^{\prime}\right\|^{2}-c_{2} b\left(w^{\prime}\right)^{q / 2} \\
& \geq\left(1-\frac{\mu_{0}}{\mu_{1}}-c_{2} b\left(w^{\prime}\right)^{(q / 2)-1}\right) b\left(w^{\prime}\right) \quad \text { for } a(y) \leq \rho^{2} \text { and } b(w) \leq \rho^{2},
\end{aligned}
$$

where $\mu_{1}$ is the next eigenvalue of $B$ after $\mu_{0}$. If we reduce $\rho$ accordingly, we can find a positive constant $v$ such that

$$
G(y, w) \geq v b\left(w^{\prime}\right), \quad a(y) \leq \rho^{2}, \quad b(w) \leq \rho^{2} .
$$

I claim that either (20) has a nontrivial solution or there is an $\epsilon>0$ such that

$$
G(y, w) \geq \epsilon \quad \text { and } \quad a(y)+b(w)=\rho^{2} .
$$


For suppose (33) did not hold. Then there would be a sequence $\left\{y_{k}, w_{k}\right\}$ such that $a\left(y_{k}\right)+b\left(w_{k}\right)=\rho^{2}$ and $G\left(y_{k}, w_{k}\right) \rightarrow 0$. If we write $w_{k}=w_{k}^{\prime}+h_{k}, w_{k}^{\prime} \in M^{\prime}$, and $h_{k} \in M_{0}$, then (32) tells us that $b\left(w_{k}^{\prime}\right) \rightarrow 0$. Thus $a\left(y_{k}\right)+b\left(h_{k}\right) \rightarrow \rho^{2}$. Since $N_{0}$ and $M_{0}$ are finite-dimensional, there is a renamed subsequence such that $y_{k} \rightarrow y$ in $N_{0}$ and $h_{k} \rightarrow h$ in $M_{0}$. By (30), $\|y\|_{\infty} \leq \delta / 4$ and $\|h\|_{\infty} \leq \delta / 4$. Consequently (29) implies

$$
2 F(x, y, h) \leq \mu_{0} h^{2}-\lambda_{0} y^{2}
$$

Since

$$
G(y, h)=b(h)-a(y)-2 \int_{\Omega} F(x, y, h) d x=0,
$$

we have

$$
\int_{\Omega}\left(2 F(x, y, h)+\lambda_{0} y^{2}-\mu_{0} h^{2}\right) d x=0 .
$$

In view of (34), this implies $2 F(x, y, h) \equiv \mu_{0} h^{2}-\lambda_{0} y^{2}$. For $\zeta \in C_{0}^{\infty}(\Omega)$ and $t>0$ small, we have

$$
2(F(x, y+t \zeta, h)-F(x, y, h)) / t \leq-\lambda_{0}\left((y+t \zeta)^{2}-y^{2}\right) / t .
$$

Taking $t \rightarrow 0$, we have $f(x, y, h) \zeta \leq-\lambda_{0} y \zeta$. Since this is true for all $\zeta \in C_{0}^{\infty}(\Omega)$, we have

$$
f(x, y, h)=-\lambda_{0} y=-A y .
$$

Similarly,

$$
2[F(x, y, h+t \zeta)-F(x, y, h)] / t \leq \mu_{0}\left[(h+t \zeta)^{2}-h^{2}\right] / t,
$$

and consequently $g(x, y, h) \zeta \leq \mu_{0} h \zeta$ and

$$
g(x, y, h)=\mu_{0} h=B h .
$$

We see from (35) and (36) that (20) has a nontrivial solution. Thus, we may assume that (33) holds.

Next, we note that there is an $\varepsilon>0$ depending on $\rho$ such that $G(0, w) \geq \varepsilon$ for $b(w) \geq \rho>0$. To see this, suppose that $\left\{w_{k}\right\} \subset M$ is a sequence such that $G\left(0, w_{k}\right) \rightarrow 0$ for $b\left(w_{k}\right) \geq \rho$. If $b_{k}=b\left(w_{k}\right) \leq C$, this implies $b\left(w_{k}\right)-\mu_{0}\left\|w_{k}\right\|^{2} \rightarrow 0$ and $\int\left[\mu_{0}-\mu(x)\right] w_{k}^{2} d x \rightarrow 0$, since

$$
G(0, w) \geq b(w)-\mu_{0}\|w\|^{2}+\int\left[\mu_{0}-\mu(x)\right] w^{2} d x \quad \text { for } w \in M .
$$

If we write $w_{k}=w_{k}^{\prime}+h_{k}$ and $w_{k}^{\prime} \in M^{\prime}$ and $h_{k} \in M_{0}$ as before, then this tells us that $b\left(w_{k}^{\prime}\right) \rightarrow 0$. Since $M_{0}$ is finite-dimensional, there is a renamed subsequence such that $h_{k} \rightarrow h$. But the two conclusions above tell us that $h=0$. Since $b(h) \geq \rho$, we 
see that $\varepsilon>0$ exists for any constant $C$. If the sequence $\left\{b_{k}\right\}$ is not bounded, we take $\tilde{w}_{k}=w_{k} / \sqrt{b_{k}}$. Then

$$
G\left(0, w_{k}\right) / b_{k} \geq b\left(\tilde{w}_{k}\right)-\mu_{0}\left\|\tilde{w}_{k}\right\|^{2}+\int\left[\mu_{0}-\mu(x)\right]\left(\tilde{w}_{k}\right)^{2} d x .
$$

Next we note that there is a $v>0$ such that

$$
G(0, w) \geq v b(w) \text { for } w \in M .
$$

Assuming this for the moment, we see that $\inf _{B} G \geq \varepsilon_{1}>0$, where

$$
B=\left\{w \in M: b(w) \geq \rho^{2}\right\} \bigcup\left\{u=\left(s \varphi_{0}, w\right): s \geq 0, w \in M,\|u\|_{D}=\rho\right\},
$$

and $\varepsilon_{1}=\min \left\{\varepsilon, v \rho^{2}\right\}$. By (26) there is an $R>\rho$ such that $\sup _{A} G=a_{0}<\infty$, where $A=N$. By Proposition 9, $A$ and $B$ form a weak sandwich pair. Moreover, $G$ satisfies (6) with $\varepsilon_{1} \leq b_{0}$. Hence, there is a sequence $\left\{u_{k}\right\} \subset D$ such that (7) holds with $c \geq \varepsilon_{1}$. Arguing as in the proof of Theorem 10, we see that there is a $u \in D$ such that $G(u)=c \geq \varepsilon_{1}>0$ and $G^{\prime}(u)=0$. Since $c \neq 0$ and $G(0)=0$, we see that $u \neq 0$, and we have a nontrivial solution of the system (20).

It therefore remains only to prove (37). Clearly $v \geq 0$. If $v=0$, then there is a sequence $\left\{w_{k}\right\} \subset M$ such that $G\left(0, w_{k}\right) \rightarrow 0$ for $b\left(w_{k}\right)=1$. Thus there is a renamed subsequence such that $w_{k} \rightarrow w$ weakly in $M$, strongly in $L^{2}(\Omega)$ and almost everywhere in $\Omega$. Consequently

$$
\int_{\Omega}\left[\mu_{0}-\mu(x)\right] w_{k}^{2} d x \leq 1-\int_{\Omega} \mu(x) w_{k}^{2} d x \leq G\left(0, w_{k}\right) \rightarrow 0
$$

and

$$
1=\int_{\Omega} \mu(x) w^{2} d x \leq \mu_{0}\|w\|^{2} \leq b(w) \leq 1,
$$

which means that we have equality throughout. It follows that $w$ must belong to $E\left(\mu_{0}\right)$, the eigenspace of $\mu_{0}$. Since $w \neq \equiv$, we have $w \neq 0$ almost everywhere. But $\int_{\Omega}\left[\mu_{0}-\mu(x)\right] w^{2} d x=0$ implies that the integrand vanishes identically on $\Omega$, and consequently $\mu(x) \equiv \mu_{0}$, violating (28). This establishes (37) and completes the proof of the theorem.

\section{References}

[Costa 1994] D. G. Costa, "On a class of elliptic systems in $\mathbf{R}^{N}$ ", Electron. J. Differential Equations 07 (1994). MR 95e:35065 Zbl 0809.35020

[Costa and Magalhães 1994] D. G. Costa and C. A. Magalhães, "A variational approach to subquadratic perturbations of elliptic systems", J. Diff. Equations 111 (1994), 103-122. MR 95f:35082 Zbl 0803.35052

[de Figueiredo and Felmer 1994] D. G. de Figueiredo and P. L. Felmer, "On superquadratic elliptic systems”, Trans. Amer. Math. Soc. 343:1 (1994), 99-116. MR 94g:35072 Zbl 0799.35063 
[Furtado and Silva 2001] M. F. Furtado and E. A. B. Silva, "Double resonant problems which are locally non-quadratic at infinity", pp. 155-171 in Proceedings of the USA-Chile Workshop on Nonlinear Analysis (Viña del Mar, 2000), edited by R. Manasevich and P. Rabinowitz, Electron. J. Differ. Equ. Conf. 6, 2001. MR 2002g:35079 Zbl 0963.35060

[Furtado et al. 2002a] M. F. Furtado, L. A. Maia, and E. A. B. Silva, "On a double resonant problem in $\mathbb{R}^{N}$,, Differential Integral Equations 15:11 (2002), 1335-1344. MR 2003g:35064 Zbl 1034.35024

[Furtado et al. 2002b] M. F. Furtado, L. A. Maia, and E. A. B. Silva, "Solutions for a resonant elliptic system with coupling in $\mathbb{R}^{N}$ ", Comm. Partial Differential Equations 27:7-8 (2002), 15151536. MR 2003f:35076 Zbl 1016.35022

[Kelley 1955] J. L. Kelley, General topology, D. Van Nostrand, Toronto-New York-London, 1955. MR 16,1136c Zbl 0066.16604

[Li and Yang 2004] G. Li and J. Yang, "Asymptotically linear elliptic systems", Comm. Partial Differential Equations 29:5-6 (2004), 925-954. MR 2005i:35071 Zbl 02130256

[Peihao et al. 2002] Z. Peihao, Z. Wujie, and Z. Chengkui, "The existence of three nontrivial solutions of a class of elliptic systems", Nonlinear Anal. 49:3 (2002), 431-443. MR 2003b:35057 Zbl 1030.35047

[Schechter 1992] M. Schechter, "A generalization of the saddle point method with applications", Ann. Polon. Math. 57:3 (1992), 269-281. MR 94c:58028 Zbl 0780.35001

[Schechter 1993] M. Schechter, "New saddle point theorems", pp. 213-219 in Generalized functions and their applications (Varanasi, 1991), edited by R. S. Pathak, Plenum, New York, 1993. MR 94i:58034 Zbl 0846.46027

[Schechter 1998] M. Schechter, "Infinite-dimensional linking”, Duke Math. J. 94:3 (1998), 573595. MR 99h:58034 Zbl 0953.58009

[Schechter 1999] M. Schechter, Linking methods in critical point theory, Birkhäuser, Boston, 1999. MR 2001f:58032 Zbl 0915.35001

[Schechter 2008] M. Schechter, "Sandwich pairs in critical point theory", Trans. Amer. Math. Soc. (2008). To appear.

[Schechter and Zou 2003] M. Schechter and W. Zou, "Weak linking", Nonlinear Anal. 55:6 (2003), 695-706. MR 2005d:58021 Zbl 1036.58010

[Silva 1991] E. A. d. B. e. Silva, "Linking theorems and applications to semilinear elliptic problems at resonance", Nonlinear Anal. 16:5 (1991), 455-477. MR 92d:35108 Zbl 0731.35042

[Silva 2001] E. A. B. Silva, "Nontrivial solutions for noncooperative elliptic systems at resonance", pp. 267-283 in Proceedings of the USA-Chile Workshop on Nonlinear Analysis (Viña del Mar, 2000), edited by R. Manasevich and P. Rabinowitz, Electron. J. Differ. Equ. Conf. 6, 2001. MR 2001j:35097 Zbl 0963.35061

[Tintarev 1999] K. Tintarev, "Solutions to elliptic systems of Hamiltonian type in $\mathbb{R}^{N}$ ”, Electron. J. Differential Equations 29 (1999). MR 2000i:35038 Zbl 0928.35043

[Zou 2001] W. Zou, "Multiple solutions for asymptotically linear elliptic systems", J. Math. Anal. Appl. 255:1 (2001), 213-229. MR 2001k:35075 Zbl 0989.35049

Received May 8, 2007. Revised November 19, 2007.

\author{
MARTIN SCHECHTER \\ DEPARTMENT OF MATHEMATICS \\ UNIVERSITY OF CALIFORNIA AT IRVINE \\ IRVINE, CA 92697-3875 \\ UNITED STATES \\ mschecht@math.uci.edu
}

